\title{
A compensation for field expansion caused by moving forward
}

\author{
HANS WALLACH and EUGENIE WALSH FLAHERTY \\ Swarthmore College, Swarthmore, Pennsylvania 19081
}

\begin{abstract}
When an object increases in size and its retinal image expands, it is perceived to grow. But image expansion caused by one's approaching an object of constant size does not result in perceived growth of the object. This is due, in part, to correct size perception which takes the distance of the object into account. But perceived growth may have another component, the perceived expanding motion of the object's contours. Failure of growth to be perceived when the image expansion is eaused by approaching an object may, in addition, be the result of a compensating process that prevents expanding motion to be perceived when the image expansion occurs during a subject's forward movement. That such a compensating process operates was demonstrated in an indirect manner. We made use of the fact that prolonged exposure of a retinal area to the same motion process leads to decrease in the speed of the resulting perceived motion and to motion aftereffect. When a compensating process operates, it might have an effect on these two consequences of motion perception, and such a result was obtained. Under conditions that would bring the compensation into effect, namely, when prolonged exposure to an expanding motion occurred only during the subject's forward movements, subsequent speed decrease was significantly diminished and motion aftereffects occurred substantially less often than in the control conditions.
\end{abstract}

During the course of bodily movements, a variety of visual stimulation is produced that results from various displacenents between the environment and our eyes. But none of these displacements give rise to motion or to other changes in the perceived environment, which, rather, remains stable and rigid. This is the result of a number of compensating processes in which the visual inputs that result from environmental displacements are more or less accurately matched up with proprioceptive information about those of our movements that caused the particular displacements. Much work is going into the investigation of those compensating processes which deal with the field displacements caused by head turning and nodding (the constancy of visual direction, see Wallach, Yablick, \& Smith, 1972). That we usually do not see objects rotate as we move past them also results from a compensating process, as Wallach. Stanton, and Becker (1974) demonstrated. Finally, as we move forward, the retinal projection of the array of objects in front of us expands. Undoubtedly, size constancy prevents this expansion from causing changes in perceived size: various cues for object distances are given along with the growing image sizes, resulting in correct size perception, and this means that sizes remain unchanged.

There are two conditions under which image expansion occurs while the subject is not moving. Either an object is moving toward the subject or a figure in a fixed position expands rapidly. When an

This work was supported by Grant $25.000 \mathrm{X}$ from the National Science Foundation to Swarthmore College, Hans Wallach, principal investigator. object is moving toward the subject, cues for the changing object distance are given along with the expansion of the object's image, and, again, size constancy prevents this expansion from causing changes in perceived size of the object. Why, under these conditions, the approaching object is sometimes perceived to expand is not a topic of this report. Neither is the question at issue of whether the expansion of the retinal image serves here as a cue for the changing distance of the object.

What concerns us here is what happens when a figure rapidly expands while both it and the subject remain in tixed positions. Here the resulting experience of growing is as much a motion in all directions as a size change. If it is true that image expansion normally causes perception of an expanding motion in addition to its effect in size perception, not seeing objects grow when we move forward suggests that a compensating process concerned with motion perception is here in operation also. To show that this is true is the purpose of the work to be reported.

It seemed to us that the method by which the operation of the other compensation processes mentioned had been demonstrated was not likely to succeed here. That method had consisted in a psychophysical procedure that measured the accuracy with which the particular compensating process functioned (Wallach \& Kravitz, 1968; Wallach, Stanton. \& Becker, 1974).In the present case, the method would have required subjects to judge the presence or absence of slight expanding or contracting motion. We were afraid that subjects would confound such motions with perceived size changes. We, 
therefore, decided to try to demonstrate the operation of such a compensation process by an indirect method.

A prolonged exposure of a retinal area to the same motion process has two consequences: One is the well-known aftereffect of motion. The other is a decrease in the speed of the perceived motion. The latter fact was reported by Gibson (1937) in connection with his theory of normalization and has been demonstrated by Carlson (1962) and by Goldstein (1957). Since speed decrease and motion aftereffect result from prolonged exposure to motion stimulation, we assumed that a compensation process that prevents or restricts motion perception would also prevent or restrict speed decrease and motion aftereffects. Therefore, if subjects were exposed to motion stimulation only under conditions that bring the compensation process into effect, speed decrease might not take place or be less than normal, and the incidence of motion aftereffect might be diminished. Our plan, thus, was to expose a stationary subject to an objectively expanding pattern under conditions that cause speed decrease and formation of a motion aftereffect. After such results had been obtained, the same sort of exposure to an expanding pattern would take place solely during the subject's forward movements, bringing compensation into action, with the expected effects on speed decrease and aftereffect formation.

The question arose as to what kind of forward movement to use. We tried walking, with little success, and ended up by using forward rocking while the subject was seated. This makes sense when one considers that, in approaching an object by a given amount, image growth is inversely proportional to the square of the object's distance from the eyes. Hence, movements that occur often when nearby objects are viewed might instigate the most effective compensation, and this is true of rocking. By effectiveness of compensation, we mean that the compensation associated with a forward movement will prevent a rather rapid image expansion from being perceived as object growth. We needed a highly effective compensation, because it had to have an effect on an image expansion caused by an objectively expanding pattern.

Rocking has, of course, the disadvantage that the eyes move forward only half the time. This made it necessary to restrict exposure to the expanding pattern to the periods of forward movement of the rocking cycle. The discontinuous exposure that this involves raised the question of whether intermittent exposure to a moving pattern can cause speed decrease and aftereffect formation. We found that it does, but that much longer exposure periods are needed than are normally required to produce these effects.
Finally, we had to face the possibility that any movement on the part of the subject during stimulation by image expansion might prevent speed decrease and motion aftereffects from developing. not solely forward movement. This called for a condition in which the expanding pattern was visible only during the backward movement of the rocking cycle.

Each of our experiments consisted. therefore, of three kinds of exposure:

Condition $F$ : The subject was rocking back and forth; the expanding pattern was visible only during the forward movement.

Condition B: The subject was rocking; the expanding pattern was seen only during the backward movement.

Condition ST: Here, too, the expanding pattern was alternately seen or invisible. with durations approximately the same as in Conditions $\mathrm{F}$ and $\mathrm{B}$, but the subject remained stationary throughout.

\section{EXPERIMENT I \\ The Effect of Forward Motion on Speed Decrease}

\section{Method}

Speed decrease can be measured with an arrangement in which two motion tields are presented in symmetrical positions about a fixation mark, one with standard and the other with variable motion velocity. A fter an initial match, in which the subject adjusts the variable velocity to appear equal to the speed of the standard. the standard motion is presented for some time, while the variable motion is stopped or invisible. At the end of this exposure period, another speed match is made. Since, at that point. the apparent speed of the standard is slower than it was when the initial match was made, the matching postexposure velocity is less than it was in the first match. This difference measures the speed decrease. In our experiment, two turning Plateau spirals were used to provide expanding motion fields. The expansion velocity was controlled by the rotation rate. ${ }^{1}$

Equipment. Two identical spiral disks of $25 \mathrm{~cm}$ diam were used. The spirals, black on white ground, were $1 / 2$ in. wide. One disk was mounted on a constant-speed motor that turned at $30 \mathrm{rpm}$ and developed a radial velocity of $2.25 \mathrm{~cm} / \mathrm{sec}$. The other spiral disk was mounted on a variable-speed motor whose rotation rate was controlled by the subject. ${ }^{2}$ The spiral disks were next to each other. spaced $6 \mathrm{~cm}$ apart. A small fixation light on a line between the centers of the disks and midway between them was visible during the entire experiment. When the subject was stationary, the distance of his eyes from the spiral disks was $325 \mathrm{~cm}$ and the visual angle of a disk was $4.35 \mathrm{deg}$. He saw the disks through a large transparent mirror that was turned to form an angle of $45 \mathrm{deg}$ with his line of sight. Two projectors served as sources of illumination. One projected two disks of light that coincided with the two spiral disks. The other illuminated a large white screen which the subject saw retlected in the mirror. Two microswitches were mounted on a frame that could be adjusted to the level of the subject's forehead. one in front and the other in back of the subject's head. The switches were inserted in stops that limited the subject's rocking movements and were spaced to allow for a displacement of the eyes of about $40 \mathrm{~cm}$. They operated a magnetic relay which alternately switched current to one or the other projector, so that a disk was illuminated during one phase of the rocking and the blank screen during the other phase. This arrangement served for Exposure Conditions $\mathrm{F}$ and $\mathrm{B}$. In Cond ition $\mathrm{St}$, where the subject sat still, two timers operated the projectors so that they changed every $1.4 \mathrm{sec}$, duplicating the average time our subjects took for each phase of the 
rocking movement. During the exposure period, the variable spiral was obscured.

During the test, the subject sat still and the two spiral disks were continuously visible. The velocity control for the variable disk was close to the subject, and it was easy for the subject to adjust it.

Procedure. In our first experiment, the spirals were arranged side by side. and each of our 18 subjects served in all three exposure conditions. There was an effect of exposure condition, but only the difference between the speed decreases obtained with the $\mathrm{F}$ and $\mathrm{St}$ conditions was significant. There may have been an effect of location of the disk visible during the exposure period, for the speed decrease was stronger for the nine subjects for whom the standard spiral was on the right. There was also an order effect that approached significance: the strongest speed decreases were obtained when the B or the St condition was presented first. Our next experiment was designed with these results in mind.

The spirals were arranged one straight above the other so that they were equally distributed with respect to the two cortical hemispheres; the variable was always above the standard. Three different groups of 10 subjects were used, one for each exposure condition. Because we had found in the first experiment that not all subjects developed a speed decrease in the St condition ${ }^{3}$ and wanted to eliminate those who did not, we had all subjects come twice. On the first visit. a subject was given one of the three exposure conditions, to which he was randomly assigned, and, on the later visit. every subject went through the St condition. If in the latter condition he did not show the speed decrease. his scores were eliminated.

Every experiment started with four preexposure matches. In each, the subject had to set the velocity control so that speed of expansion of the variable spiral appeared equal to that of the standard spiral. Before each match, the experimenter set the velocity alternately much lower or much higher than equality with the standard. The first two matches were for practice, and the average of the last two was used for comparison with the postexposure match. The subject was asked to keep his eyes on the tixation point, to make his match solely on the basis of the speed of the expansion and to avoid paying attention to the rotation rate. Those subjects who did not or could not follow the latter part of these instructions would in the elimination experiment show no speed decrease and would be eliminated.

In the exposure period, which followed the preexposure matches, the light beam that illuminated the variable spiral disk was stopped. The illumination of the standard disk became intermittent, alternating with the blank field. Only the fixation mark remained visible throughout, and the subject was instructed to "keep his eyes glued to it." This period lasted 10 min and thus provided a total of 5 min exposure to the expanding spiral. Because it seemed possible that the exposure effect that manifested itself in the speed decrease dissipated rapidly, there was only one postexposure match. The experimenter set the starting velocity of the variable spiral either higher or lower than the standard velocity, alternating between subjects on each exposure condition.

In order to obtain 10 subjects for each of our three conditions. 46 subjects were put through the experimental procedure; 16 subjects were eliminated because they showed no speed decrease in the elinination experiment. Eleven of the 30 subjects for whom results will be reported were college students, and the remainder were high school students.

\section{Results}

The mean pre-and postexposure speed matches for the different exposure conditions of the main experiment are listed in Table 1 . The speed changes catused by the exposure period are given in the last row, with a negative number denoting a speed decrease. The table show's that a sizable average speed decrease of $36.6 \%$ was obtained from the 10 subjects who in the main experiment had been exposed to the St condition. There was no change for the 10 subjects
Table 1

Mean Matching Velocities, Measuring the Apparent Speed of Expansion of a Spiral Disk Before and After Prolonged Exposure Under Three Conditions

\begin{tabular}{llcc}
\hline Conditions & F & B & St \\
\hline Preexposure & 2.04 & 2.49 & 2.73 \\
Postexposure & 2.04 & 2.00 & 1.73 \\
Speed Change & 0 & -.49 & -1.0 \\
\hline
\end{tabular}

Note-Standard velocity of expansion is $2.25 \mathrm{~cm} / \mathrm{sec}$.

who had been exposed to the $F$ condition, and this agreed with our expectation. The large and very significant difference between $36.6 \%$ and $0 \%$ showed that the $\mathrm{F}$ condition was highly effective in preventing speed decrease. The B condition produced a speed decrease of $19.7 \%$, which was significantly different from the corresponding value obtained for the $F$ condition $(t=2.60 ; p<.01)$. Presenting expanding motion only during forward movements thus caused a significantly smaller speed decrease than presenting it during backward movement only. Therefore, it was, though perhaps only in part, forward movement rather than movement in a more general sense that was effective in preventing speed decrease from resulting from prolonged exposure to expanding motion. The difference in the mean speed decreases obtained for the $B$ and the St conditions $(t=1.89$; $p<.05)$ approached significance. It is, therefore, possible that backwards movements also have some effect on speed decrease.

\section{EXPERIMENT II The Effect of Forward Motion on the Incidence of Motion Aftereffect}

\section{Method}

Two procedures have been used to measure the magnitude of the motion aftereffect in a manner applicable to expanding motion. compensation (Scott. Jordan. \& Powell, 1963), and measuring the duration (Bonnet \& Pouthas, 1972). We found that the compensation procedure was very difficult for a subject, and we were not convinced that duration adequately represents the magnitude of the aftereffect. Our measure was therefore the frequency with which aftereffects were reported after prolonged exposures to an expanding spiral under our three conditions.

The setup was similar to that used in Experiment 1 . A single spiral disk was mounted on a variable-speed motor. The fixation point was in the center of the spiral disk. The distance of the subject's eyes from the disk was $175 \mathrm{~cm}$ when the subject was stationary. At this distance. the disk's visual angle was $8 \mathrm{deg}$ $10 \mathrm{~min}$. Therefore, half of the diameter of the spiral's retinal image fell into the macula, while its outer ring was given in peripheral vision.

Procedure. The total exposure period always lasted $80 \mathrm{sec}$. Under all exposure conditions, the spiral disk again was visible in alternation with a blank field. We tested for an aftereffect by stopping the rotation of the spiral disk and by asking the subject to keep fixating and report what. if any, motion he saw. The rotation rate of the disk was determined individually for each subject. It was the low est velocity at which he reported an aftereffect twice in a row under the St conditions for both an expanding and a contracting spiral. This velocity was found by raising the radial velocity of the spiral in steps of $.375 \mathrm{~cm} / \mathrm{sec}$. The radial velocities so selected 
varted from . 975 to $3.0 \mathrm{~cm}$ sec with a mean of $2.1 \mathrm{~cm} / \mathrm{sec}$.

A group of 10 subjects was employed in two sessions, a week or more apart. In the first session. the subject's rotation rate was found and, after a 10-min rest period. the first part of the experiment was begun. It consisted of exposing the subject to the expanding spiral six times. twice under each of three exposure conditions and each followed immediately by the test. The three exposure conditions were: The St condition and the $F$ condition of Experiment I; in the latter. the expanding spiral was visible only during the forward phase of the subject's rocking movement. In the third condition (FC), the spiral was contracting and visible only during forward movement. In the second session. the subject was exposed twice to each of the three following exposure conditions: the St condition. Condition B of Experiment $I$, in which the expanding spiral was visible only during backward movements, and a $B C$ condition in which the contracting spiral was visible only during backward movements. Within each session, sequence effects were controlled by counterbalancing. and individual exposure and test sequences were separated by 5 -min-long rest periods.

\section{Results}

A report of apparent contraction of the stationary spiral in the tests following exposures to the St. B, and $F$ conditions was scored as an aftereffect. while tests after the FC and BC conditions required a report of apparent expansion. The number of aftereffects scored for each exposure condition is listed in Table 2. Since each of the 16 subjects was twice exposed to each condition involving rocking. the maximum number obtainable for each of the conditions was 32 . There were four exposures to the $\mathrm{St}$ condition for each subject. The number of aftereffects reported for this condition was halved before it was listed in Table 2.

That. after exposure in the stationary condition, an aftereffect was obtained almost every time is due to the fact that for every subject the expansion velocity was so selected that this would occur. But when subjects had seen the expanding spiral only during their forward movements ( $F$ condition), an aftereffect was reported only 12 times; the difference between 12 out of 32 and 31 out of 32 is significant at the .001 level. The result for the $F$ condition was also signiticantly different $(p=.003$ ) from that for the $B$ condition. where the expanding spiral was visible during backward movement only and caused an attereffect 25 times. This result corresponds to that of Experiment I, where the speed decrease measured after exposure to the $F$ condition was significantly smaller than that measured after exposure to the $B$ condition.

Table 2

Number of Aftereffects Reported After Different Exposure Conditions

\begin{tabular}{llll} 
& \multicolumn{2}{c}{ Conditions } & \\
\cline { 2 - 3 } & Movement & Spiral & N \\
\hline St & Stationary & Expanding & 31 \\
F & Forward & Expanding & 12 \\
B & Backward & Expanding & 25 \\
FC & Forward & Contracting & 28 \\
BC & Backward & Contracting & 27 \\
\hline
\end{tabular}

These two results are the principal linding of our experiments. We regard then as manifestations of a compensation for image expansion that oceurs during forward movements. This compensation is presumably learned when, under ordinary conditions, we move forward and the retinal images of objects in tromt of the eyes simultaneously expand. That the compensating process is indeed acquired is supported by a further result of Experiment II, namely that exposure to a contracting spiral solely during batchuard movements does not appreciably diminish the incidence of the aftereffect. In the BC condition, an aftereffect developed in 27 out of 32 cases. This result reflects the fact that in ordinary life moving backwards while looking straight ahead occurs less often than do forward movements.

\section{DISCUSSION}

It was reported that, in Experiment I, exposure to the expanding spiral during backward movements caused a mean speed decrease that was smaller than the speed decrease measured after an exposure during which the subject was stationary, and that this difference was almost signiticant. If this result were accepted. it would mean that with some subjects or to some degree with all subjects the compensating process of which our results are supposedly manifestations is not as specitic as the term implies. This process that prevents image expansion caused by the subject's own movements from having its normal perceptual effect presumably does this by matching up, more or less accurately, proprioceptive data about the subject's forward movements with the simultaneous image expansion. But the underlying mechanism may, to some extent. operate on backward movements also. This explanation is, however, not confirmed in Experiment II, where the two conditions in which exposure occurred during backwards movements caused little, if any, diminished incidence of aftereffects.

Other processes that compensate for changes in stimulation caused by one's own movements operate with widely differing accuracy. The compensation for field displacements due to head movements (CVD) does so very accurately, so that small objective field displacements that occur during head movements are readily perceived. The compensation for changes in the orientation of objects that we pass (Wallach. Stanton, \& Becker, 1974), on the other hand, is very inaccurate. What can we say about the compensation for tield expansion during forward movements? What evidence we have seems to be contradictory. That there were, in Experiment II, 12 reports of aftereffects in the $F$ condition indicates that there was not always compensation for an expansion that greatly execeded the normal expansion of the spiral disk due to forward movement. As stated above, the mean 
cxpansion rate of the spiral due to rotation was $2.1 \mathrm{~cm}$ sec. While the average expansion rate of the spiral disk duc to the subject's forward movement amounted to only $1.0 \mathrm{~cm} / \mathrm{sec}^{5}$ On the other hand, compensation appears to have been complete in the $F$ condition in Experiment I. But with only 10 subjects involved in these results, the confidence limits of this result $( \pm 14 \%)$ are so large that such a reading of the result is not justitied.

It should be made clear, at any rate, that if the compensation for image expansion during forward movement operates with some degree of accuracy, the primary distance between the eyes and the object catusing the image in question, that is, the distance that is altered by the subject's movement, must also be taken into account. This is necessary because the amount of image expansion caused by a certain distance change depends strongly on that primary distance, on the primary distance squared, to be exact. Hence, a compensating process that operates quantitatively must take primary distance into account in addition to the distance change. The operation of a tripartite process of such complexity had been demonstrated in the constancy of visual direction, where the rate of object displacement catused by head turning also depends on a third variable, the object distance. Hay and Sawyer (1969) and Wallach, Yablick, and Smith (1972) demonstrated that object distance is, indeed, taken into account in the constancy of visual direction.

\section{REFERENCES}

Bonnet, C., \& Pouthas, V. Interactions between spatial and kinetic dimensions in movement aftereffect. Perception \& Psichophisics, 1972, 12, 193-200.

Carlson. V. R. Adaptation in the perception of visual velocity. Journal of Experimental Psichology. 1962. 64. 192-197.

Gibson, J. Adaptation with negative aftereffect. Psychological Review, 1937, 44, 222-244

Goldstein, A. G. Judgements of visual velocity as a function of length of observation time. Journal of Experimental Psychology. 1957. 54, 457-461.

HAY, J. C.. \& SAWYER, S. Position constancy and binocular convergence. Perception \& Psychophysics, 1969, 5, 310-312.

Scott. T. R., Jordan, A. E., \& Powell, D. A. Does the visual aftereffect of motion add algebraically to objective motion of the test stimulus? Joumal of Experimental Psychology, 1963, 66, $500-505$.

Wallach. H., \& Kravitz. J. H. Adaptation in the constancy of visual direction tested by measuring the constancy of auditory direction. Perception \& Psychophysics, 1968, 4, 299-303.

Wallach, H., Stanton, L., \& Becker. D. The compensation for movement-produced changes of object orientation. Perception \& Psychophysics, 1974, 15, 339-343.

Wallach, H., Yablick, G. S., \& Smith, A. Target distance and adaptation in distance perception in the constancy of visual direction. Perception \& Psychophysics, 1972, 12. 139-145.

\section{NOTES}

1. An expanding Plateau spiral does not correctly duplicate the expansion of the retinal projection of a pattern that is approached. Because approach causes proportional size increase, points near the center of the expanding image are less rapidly displaced than more eccentric points. The Plateau spiral, on the other hand, is so constructed that the rate of displacement in the radial directions is the same everywhere.

2. A de shunt motor was used, whose velocity was varied by changing the armature voltage. This arrangement yields a linear relation between armature voltage and rotation velocity.

3 . This does not mean that there are numerous persons who do not develop speed decrease. It should be remembered that in our experiments subjects were exposed to the expanding spiral only intermittently.

4. As noted, two different procedures were employed in the postexposure matches: in each of the three conditions, the starting velocity of the variable spiral was, for half the subjects. higher than the velocity of the standard, and for the other half, it was lower. An analysis of variance was performed to compare the importance of these initial velocity settings with the effect of the experimental conditions. It showed that the velocity settings had no significant effect $(F=2.12$, $\mathrm{df}=1,24 ; \mathrm{p}<.25)$ as compared with the effect of the conditions $(\mathrm{F}=8.99$, $\mathrm{df}=2,24 ; \mathrm{p}<.01)$.

5 . The reason for computing an average rate for the expansion due to the subject's forward movement is given in Note 1.

(Received for publication October 11, 1974; revision received January 8,1975 .) 